\title{
An Initial Investigation of Parenting Stress, Social-Emotional Protective Factors, and Behavior Concerns within a Head Start Population
}

\author{
Stacy L. Bender ${ }^{1} \&$ John S. Carlson ${ }^{1}$ \\ ${ }^{1}$ Department of Counseling, Educational Psychology, and Special Education at Michigan State University, East \\ Lansing, MI, USA \\ ${ }^{2}$ University of Rochester Medical Center, Rochester, NY, USA \\ Correspondence: Stacy L. Bender, University of Rochester Medical Center, Rochester, NY 14642, USA. Tel: \\ 1-585-276-4201. E-mail: Stacy_Bender@urmc.rochester.edu
}

Received: June 15, 2012 Accepted: January 24, 2013 Online Published: March 21, 2013

doi:10.5539/jedp.v3n1p113 URL: http://dx.doi.org/10.5539/jedp.v3n1p113

\begin{abstract}
This study examines associations between parenting stress, protective factors, and behavior concerns in an at-risk preschool population. Participants included 77 parents of children enrolled in Head Start programs in the Midwest, which served predominantly low-income, diverse parent and child populations. Parents completed a parenting stress questionnaire and a questionnaire that assessed for children's protective factors and behavior concerns. Associations were found between elevated protective factors and low parenting stress, as well as between elevated behavior concerns and elevated parenting stress. Parents of children exhibiting more protective factors had significantly less parenting stress, compared to parents of children exhibiting more behavior concerns. Additionally, parents with non-clinical levels of parenting stress had children that exhibited significantly more protective factors and less behavior concerns compared to children of parents with clinically significant levels of parenting stress. This is the first study to demonstrate that low levels of parenting stress are associated with more protective factors in an at-risk preschool population. Future research needs to explore the predictive nature parenting stress and protective factors have on one another, given the bi-directionality of parent-child interactions.
\end{abstract}

Keywords: parenting stress, protective factors, behavior problems, head start

\section{Introduction}

Parenting stress is defined as the aversive psychological reaction to the demands of being a parent and are perceptions made by the parent in the context of the parenting role. Parenting stress is often created when there is a discrepancy between the perceived demands of parenting and the resources available to meet those demands (Abidin, 1995). It is represented by a process linking a) the task demands of parenting, b) the parent's psychological well-being, c) parenting behavior, d) qualities of the parent-child relationship, and e) the child's psychological adjustment. This can take the form in parents' feelings of low efficacy and competence to parent their child as well as negative perceptions of their child. It has been suggested that these feelings and perceptions are associated with an increased likelihood of poor parenting behaviors, which may lead to negative child behavior, thus exacerbating negative perceptions and elevating stress. Given this, convincing evidence has been provided to suggest that parenting stress is a risk factor for the development and/or maintenance of behavior problems (Qi \& Kaiser, 2003).

It is natural for all parents to experience some level of parenting stress, however when it is elevated and chronic, negative outcomes are more likely to occur (Deater-Deckard, 1998). It is important to examine parenting stress given that it influences both parent and child behavior (Abidin, 1995). Parenting stress has been linked to less optimal parent-child interactions, insecure child attachments, and child abuse and neglect (e.g., Crnic, Gaze, \& Hoffman, 2005; Guterman, Lee, Taylor, \& Rathouz, 2009). Research has also indicated that parenting stress is associated with children's behavior problems. Barry and colleagues (2005) explored factors associated with disruptive child behavior. Through regression analyses, researchers found that parenting stress was significantly related to maternal reports of inattention, aggression, and hyperactivity. Interestingly, parenting stress was not 
significantly related to teacher rated behavior problems. The researchers suggested that this could be due to maternal bias. Additionally, the behavior children exhibited and the perceptions of parenting stress is likely a bidirectional relationship, where one exacerbates the other.

Studies have suggested that externalizing behavior problems predict initial status of parenting stress and changes in levels of parenting stress over time (Williford, Calkins, \& Keane, 2007). In a longitudinal study, conducted by Williford and colleagues (2007), children's behavior was assessed at ages 2, 4, and 5 years of age and maternal reports of parenting stress were collected. This is one of the few studies to examine the stability of parenting stress over time and one of the first to investigate the causal mechanisms of parenting stress. Hierarchical linear modeling was used to examine the stability of stress by examining factors that predicted the initial status of elevated stress at age 2, and whether parenting stress increased or decreased over time. Results suggested that behavior problems predicted the initial status of parenting stress and also predicted an elevation or decline in stress over time.

Not only have children's behavior problems predicted parenting stress, but parenting stress has also been found to predict children's behavior (Shaw, Winslow, Owens, \& Hood, 1998). In another longitudinal study, Shaw and colleagues (1998) collected data on infants and continued to collect data until they were 3 and half years of age. The purpose of the study was to examine the relationship between multiple family stressors at the ages of 18 and 24 months and children's behavior at 24 and 42 months, both individually and cumulatively. In this study, parenting stress was found to be related to the children's current behavior problems, with elevated parenting stress predicting more behavior problems, and less parenting stress predicting fewer behavior problems. Parenting stress was also a consistent predictor of children's behavior at both 24 and 42 months.

Children and their families attending Head Start are considered at-risk given their low-income status. Parents with low socioeconomic status (SES) may experience parenting stress more frequently given their limited financial resources, which may produce additional stress (Linver, Brooks-Gunn, \& Kohen, 2002). Additionally, low SES is also related to the development of social-emotional and behavioral problems in children (Qi \& Kaiser, 2003). Prevalence rates of children exhibiting behavior problems from low-income families range from $16 \%$ to $31 \%$ compared to $3 \%$ o $6 \%$ of the children in the general population (Qi \& Kaiser, 2003). Families from low SES backgrounds are more likely to experience disruptions in parent-child relationships and exhibit poorer parenting practices (Conger et al., 2002; Mistry, Vandewater, Huston, \& McLoyd, 2002).

These negative outcomes for children and parents as a result of behavior problems, parenting stress, and poverty illustrate the transactional nature of parent-child interactions. Parenting stress may lead to the development or maintenance of behavior problems given that children's development is the product of dynamic, transactional relationships between characteristics of the child, parents, and environment (Sameroff \& Chandler, 1975). Children's disruptive behavior may exacerbate parenting stress, which may lead to less optimal parent-child interactions and in turn, poor interactions may lead to increased behavior problems. The compounded risk of low SES may increase the likelihood of these negative outcomes. The evidence discussed prior suggests a link between parenting stress and children's behavior problems and parenting stress and low SES. However, no examination of children's protective factors, competence, or prosocial behaviors were examined in these studies.

Although undesirable factors such as parenting stress and behavior problems have the potential to negatively impact the parent-child interaction, protective factors may have the potential to positively impact the interaction. Protective factors are individual or environmental characteristics that promote or maintain healthy development and moderate or buffer the negative effects of stress (Rutter, 1979). Individual protective factors include behaviors such as self-control, initiative, attachment, and the ability to communicate effectively (LeBuffe \& Naglieri, 1999). Children exhibiting these behaviors are more likely to have stronger relationships with parents, childcare providers and teachers. Children exercising self-control and initiating behaviors toward peers are also more likely to be accepted by their peers (Raver, Blackburn, Bancroft, \& Torp, 1999). These positive responses from peers and adults have been suggested to lead to more positive outcomes for children, including higher academic achievement and stronger relationships with peers and teachers. Given this, it is important to examine the association between protective factors and parenting variables, such as parenting stress. Positive behaviors exhibited by children and lower stress experienced by parents could serve as protective factors for both children and parents.

While examining behavior problems and assessment tools examining behavior problems in children has been much more common than examining protective factors, increasing research has begun assessing protective factors in young children as well. As the investigation of protective factors grows, the development of measures assessing protective factors also continues to grow. The Devereux Early Childhood Assessment (DECA) is one 
strengths-based assessment for preschool-aged children (LeBuffe \& Naglieri, 1999). The DECA has been used to evaluate protective and risk factors within Head Start populations. For example, Brinkman and colleagues (2007) investigated parental reports of behavior and found that two cohorts of children in a Head Start sample were more likely to exhibit fewer protective factors and more behavioral concerns, compared to the DECA norms reported in the manual. These findings are consistent with past literature, which suggests children living in poverty tend to exhibit more behavior problems and fewer protective factors.

Although there has been an increased awareness that parenting stress is a risk factor for the development of behavior problems in young children, research examining the association between low levels of parenting stress and high levels of protective factors in children is limited, especially in low-income families. Parenting stress may impact child development given the bidirectional nature of parent-child relationships. Examining the association between parenting stress and protective factors in low-income families is important give that poverty status is a risk factor for the development of behavior problems. Children experiencing multiple risk factors have an increased risk for the development of behavior problems therefore investigating ways in which to increase protective factors for at-risk children may result in buffering of negative behavioral outcomes. Since much literature suggests that poverty and parenting stress are risk factors for the development of behavior problems in children, parenting stress should be further investigated to better understand its association to protective factors, in hopes to reduce the accumulation of risk factors experienced by the child. This exploratory study examines whether associations exist between parenting stress, protective factors, and behavior concerns within an at-risk preschool population by investigating the following questions:

1) Is parenting stress associated with children's protective factors and behavior concerns?

2) Do parents of children with high protective factors and parents of children with high behavior concerns exhibit significant differences in parenting stress?

3) Do parents with clinically significant levels of parenting stress and parents with non-clinical levels of parenting stress have children that exhibit significantly different protective factors and behavior concerns?

\section{Method}

\subsection{Participant Characteristics}

The participants consisted of 77 parents of children attending various Head Start programs. Most of the parents recruited for the study were mothers $(90 \%)$. Other raters included fathers $(5 \%)$, grandparents $(4 \%)$, or other adults (1\%). Overall, $95 \%$ of the raters were female and $5 \%$ of the raters were male. Sixty percent of the sample was European American, 16\% were African American, 10\% were Hispanic, 4\% were categorized as "other", and $10 \%$ preferred not to answer. The age range of participants was between 21 to 64 years of age. The mean age of participants was 31 years.

The marital status of the majority of the sample was married (40\%) or single (35\%). The marital status of others in the sample included separated (5\%), divorced (8\%), engaged (4\%), or living with boyfriend or girlfriend (8\%). In regards to highest level of educational attainment of the sample, $28 \%$ received a high school diploma/GED, 45\% completed some college, $8 \%$ received an Associate's Degree, 9\% received a Bachelor's Degree, 3\% received an Advanced Degree, and 7\% did not yet complete high school. Approximately half of the sample reported that their household income level in the past 12 months was less than $\$ 15,000$ (46\%). Thirty-one percent of the sample reported a household income level of $\$ 15,000$ to $\$ 34,999,9 \%$ reported $\$ 35,000$ to $\$ 54,999$, $6 \%$ reported $\$ 55,000$ to $\$ 74,999$, and $8 \%$ preferred not to respond.

Of the children rated by participants, $56 \%$ were girls and $44 \%$ were boys. Children rated were between the ages of 36 to 62 months. The mean age of children was 50 months.

\subsection{Procedure}

Data was collected from parents at various Head Start programs in the Midwest, which served predominantly low-income, diverse parent and child populations. Following the university's Institutional Review Board approval and agreement of Head Start programs, a first wave of 550 recruitment fliers was distributed to approximately 45 classrooms from 13 programs. Fliers were placed in Head Start teachers' mailboxes and were asked to distribute to parents of children in their classes. From this wave, 25 parents were recruited (4.5\%). Approximately two months later, a second wave of 550 fliers were distributed to the same teachers' mailboxes. From this second wave, ten parents were recruited $(1.8 \%)$. A third wave of 550 fliers was distributed to approximately 30 classrooms from 19 additional programs and $42(7.5 \%)$ additional parents $(7.5 \%)$ were recruited. An overall total of 1,650 fliers were distributed to approximately 75 classrooms from 32 programs. A total of 77 parents were recruited (4.7\%) to comprise the study sample. Information was not collected regarding 
which programs their child attended.

Recruitment fliers distributed to participants included a short description of the study and the researcher's contact information. Interested participants $(n=90)$ contacted the researcher via telephone or email, with the majority of parents contacting the researcher via telephone $(n=80)$. The researcher explained to interested parents what was to be expected if they participated in the study. If participants did not provide a mailing address in their phone message or email, the researcher was required to call or email the participants. Of the 90 participants that contacted the researcher, two participants could not be reached via telephone to provide the researcher with a mailing address. All parents $(n=88)$ that contacted and spoke with the researcher indicated that they were still interested after the description of the study was provided. The researcher sent a packet in the mail to the interested participants. Of the 88 participants that were sent a packet, 77 participants completed and returned the packet to the researcher.

The researcher constructed a packet consisting of two consent forms, one for participants to keep for their records and one to be signed and returned to the researcher, the Devereux Early Childhood Assessment (DECA), the Parenting Stress Index-Short Form (PSI-SF), and a self-addressed, stamped envelope. Participants indicated their voluntary participation by mailing their signed consent and completed packet to the researcher. After the researcher received the signed consent form and two completed questionnaires, participants received a $\$ 10$ gift card for a local grocery and retail store in the mail. Participants were asked to sign a receipt, indicating that they received the gift card, and mail the signed receipt to the researcher in the self-addressed, stamped envelope provided.

Follow-up phone calls were conducted if the researcher did not receive participants' packets two weeks after they were mailed. Of the interested participants that were sent packets by the researcher $(n=88), 87 \%$ of participants $(N=77)$ returned completed packets. Follow-up phone calls were also conducted if the researcher did not receive the signed gift card receipt.

\subsection{Measures}

\subsubsection{The Devereux Early Childhood Assessment}

The Devereux Early Childhood Assessment (DECA; LeBuffe \& Naglieri, 1999) is a nationally, norm-referenced, strength-based assessment that evaluates protective and risk factors in preschool children. The DECA consists of 37 items; 27 items assess Total Protective Factors (TPF) and 10 items assess for Behavior Concerns (BC). The TPF scale provides an indication of behavioral strengths and resilience, whereas the BC scale measures behavioral problems young children may exhibit including aggression, withdrawal, attention, and dysregulated emotions. The 27-item TPF score is compromised of three subscales including Initiative, Self-Control, and Attachment. Initiative measures a child's ability to independently think, select choices, and engage in actions that will help meet their needs. An example question on this subscale is "During the past 4 weeks, how often did the child do things for himself/herself?" The Self-Control subscale examines a child's ability to appropriately express their feelings using words and actions. An example of a question from this subscale is "During the past 4 weeks, how often did the child control his/her anger?" The third subscale on the TPF scale, Attachment, evaluates a child's bond with significant caregivers, such as their parents. An example of a question assessing for Attachment is "During the past 4 weeks, how often did the child show affection for familiar adults?"

The items were assessed on a Likert scale, with five possible responses, ranging from never (0) to very frequently (4). On the DECA, children can receive ratings of "strength" $(t \geq 60)$, "typical" $(40 \leq t \leq 60)$, or "concern" $(t \leq 40)$ on the TPF scale and TPF subscales. On the BC scale, children can receive ratings of "strength" $(t \leq 40)$, "typical" (40 $\leq t \leq 60)$, or "concern" $(t \geq 60)$. Given this, ratings of $t \geq 60$ were considered "high" scores for the TPF scale and TPF subscales. However, ratings of $t \geq 65$ were considered "high" scores for the BC scale in the current study. One and a half standard deviations (rather than one standard deviation) above the mean were used as the cut-off to capture children who were exhibiting more severe behavior problems. The standardization sample consisted of 2,000 preschool children and accurately reflects the diversity of preschool children in the United States. The authors of the DECA report that the instrument has adequate psychometric properties. Internal reliability alpha coefficients for the scales based on parent ratings are: .84 (Initiative), .86 (Self-control), .76 (Attachment), .91 (Total Protective Factors), and 71 (Behavior Concerns) (Lebuffe \& Naglieri, 1999).

Relatively few studies have utilized the DECA within a Head Start population, yet supportive evidence for using the DECA within this population exists (Brinkman et al., 2007). As mentioned previously, Brinkman and colleagues (2007) have examined behavioral risk and protective factors in two cohorts of Head Start children. Results suggested that the cohorts of Head Start children exhibited more behavioral concerns and less total protective factors in both cohorts compared to the national DECA norms. Given that the results were consistent 
with previous literature, the researchers suggested that the DECA is a reliable measure of accurately identifying risk and protective factors within a population considered behaviorally at risk.

\subsubsection{Parenting Stress Index-Short Form}

The Parenting Stress Index-Short Form (PSI-SF $3^{\text {rd }}$ Ed.; Abidin, 1995) is an early identification assessment of parenting and family characteristics that fail to promote normal development and functioning in children, children with behavioral and emotional problems, and parents who are considered at-risk for dysfunctional parenting. The PSI-SF is a derivative of the full length test, the Parenting Stress Index (PSI $3^{\text {rd }}$ Ed.; Abidin, 1995). The PSI-SF consists of 36 items. The Total Stress score is comprised of three subscales, Parental Distress, Parent-Child Dysfunctional Interaction, and Difficult Child. The items were assessed on a Likert scale, with five possible responses ranging from strongly disagree (1) strongly agree (5). The PSI Parental Distress Domain correlates .92 with the PSI-SF Parental Distress subscale and the PSI Child Domain correlates .87 with the PSI-SF Difficult Child subscale. The PSI-SF Parent-Child dysfunctional Interaction subscale was derived from the PSI Child and Parent domains. The PSI-SF Parent-Child Interaction subscale correlates .73 with the PSI Child domain and .50 with the Parent domain. The PSI Total Stress scores correlates .95 with the PSI-SF total. The PSI-SF has been empirically validated to predict observed parenting behavior as well as children's current and future behavioral and emotional adjustment (Abidin, 1995).

The PSI-SF has also been examined within low-income, predominately minority populations. The psychometric characteristics of the questionnaire as well as the relations between the PSI-SF, demographics, and psychosocial factors associated with parenting stress with mothers of children who attended Head Start have been examined (Reitman, Currier, \& Stickle, 2002). Results indicated that internal consistencies were very good to excellent and were similar to those reported in the PSI professional manual. Furthermore, mothers' reports of psychological symptomology, parenting stress, and frequencies of child behavior problems were within a standard deviation of the normative range for these measures which suggests that mothers in the Head Start sample were representative in self-reported parenting stress, psychological symptomology, and ratings of their children's behavior. Additionally, the Parental Distress and Parent-Child Dysfunctional Interaction subscales have been extensively examined with parents of children attending Early Head Start and were found to be both reliable and valid (Whiteside-Mansell et al., 2007). These findings support the use of the PSI-SF with populations including low-income, racially diverse parents.

The DECA and the PSI-SF appear to be reliable measures for identifying risk and protective factors and parenting stress levels for the sample in the current study. Internal reliability coefficients of the DECA were high. Coefficients of the Total Protective Factors and Behavior Concerns scales were .92 and .76 respectively. These coefficients were slightly higher than coefficients reported in the DECA manual. The internal reliability coefficient for the PSI-SF was also high. The coefficient for the PSI-SF Total Score was .94. This coefficient was similar to the coefficient reported in the PSI manual.

\subsection{Data Analysis}

Correlation analyses were conducted to investigate whether parenting stress experienced by parents was associated with more protective factors or behavior concerns. Analysis of variance (ANOVA) procedures were conducted to assess whether parents of children with more protective factors and parents of children with more behavior problems exhibited significantly different levels of parenting stress. ANOVA procedures were also conducted to assess whether clinical or non-clinical levels of parenting stress were associated with protective factors and behavior concerns. The Statistical Package for the Social Sciences (SPSS) was used to conduct all of the analyses.

\section{Results}

\subsection{Descriptive Statistics}

Overall, children had mean $t$-scores for TPF and TPF subscales that were within the typical range. The BC mean $t$-score was slightly higher than the mean for TPF and TPF subscales, however the score was still considered to be in the typical range. Parents' PSI-SF Total Stress mean score were also in the normal range (Table 1).

\subsection{Correlation Analyses}

Significant correlations were found between the PSI-SF, TPF, TPF subscales, and BC scores (Table 2). A significant negative correlation was found between the Parenting Stress Total score and children's TPF score, indicating that more protective factors were related to less parenting stress. Similarly, the Parenting Stress Total Score and TPF subscales indicated significant negative correlations for Attachment, Self-Control, and Initiative. Results for TPF and Parenting Stress subscales revealed significant negative correlations for Parental Distress, 
Difficult child, and Parent-Child Dysfunctional Interaction. More

Table 1. Means and standard deviations for the Devereux Early Childhood Assessment and the Parenting Stress Index-Short Form

\begin{tabular}{llll}
\hline Subscale & M & SD & Range \\
\hline DECA (T-Score) & & & \\
Total Protective Factors & 50.0 & 10.3 & $28-72$ \\
$\quad$ Girls & 51.4 & 9.9 & $34-72$ \\
Boys & 48.2 & 10.7 & $28-72$ \\
& & & \\
Attachment & 49.8 & 11.3 & $28-72$ \\
Girls & 49.2 & 11.0 & $30-72$ \\
Boys & 50.5 & 11.9 & $28-72$ \\
& & & \\
Self-Control & 51.3 & 10.5 & $28-72$ \\
Girls & 52.0 & 9.8 & $31-70$ \\
Boys & 50.5 & 11.5 & $28-72$ \\
& & & \\
Initiative & 51.1 & 10.5 & $30-72$ \\
Girls & 53.6 & 9.3 & $34-72$ \\
Boys & 49.2 & 11.0 & $30-72$ \\
& & & \\
Behavior Concerns & 57.8 & 9.9 & $28-72$ \\
Girls & 57.3 & 9.5 & $35-72$ \\
Boys & 58.4 & 10.5 & $28-72$ \\
& & & \\
PSI-SF (Raw Score) & 76.4 & 22.5 & $36-139$ \\
Total Score & 76.1 & 21.4 & $47-134$ \\
Girls & 24.1 & $36-139$ \\
Boys & & &
\end{tabular}

Table 2. Correlations between protective factors, behavior concerns, and parenting stress

\begin{tabular}{|c|c|c|c|c|c|c|c|c|c|}
\hline Variable & 1 & 2 & 3 & 4 & 5 & 6 & 7 & 8 & 9 \\
\hline 1. DECA Total Protective Factors & --- & & & & & & & & \\
\hline 2. DECA Initiative & $.93 * *$ & --- & & & & & & & \\
\hline 3. DECA Self-Control & $.81 * *$ & $.63 * *$ & --- & & & & & & \\
\hline 4. DECA Attachment & $.76^{* *}$ & $.62 * *$ & $.43^{* *}$ & --- & & & & & \\
\hline 5. DECA Behavior Concerns & $-.45 * *$ & $-.40 * *$ & $-.48 * *$ & $-.28 * *$ & --- & & & & \\
\hline 6. PSI-SF Parenting Stress & $-.68 * *$ & $-.54 * *$ & $-.63 * *$ & $-.58 * *$ & $.56^{* *}$ & --- & & & \\
\hline 7. PSI-SF Parental Distress & $-.48 * *$ & $-.38 * *$ & $-.45^{* *}$ & $-.51 * *$ & $.42 * *$ & $.84 * *$ & --- & & \\
\hline 8. PSI-SF Difficult Child & $-.64 * *$ & $-.51 * *$ & $-.68 * *$ & $-.51 * *$ & $.56^{* *}$ & $.90 * *$ & $.58^{* *}$ & --- & \\
\hline 9. PSI-SF Parent-Child Dysfunctional Interaction & $-.63 * *$ & $-.60 * *$ & $-.49 * *$ & $-.54 * *$ & $.44^{* *}$ & $.85^{* *}$ & $.57^{* *}$ & $.72 * *$ & --- \\
\hline
\end{tabular}

$* \mathrm{p}<.05 ; * * \mathrm{p}<.01$

Protective factors were correlated with less parenting stress. A significant positive correlation was found between the Parenting Stress Total and children's BC scores, suggesting that the higher children's BC scores were, the 
more parenting stress was reported. Results for BC and Parenting Stress subscales indicated significant positive correlations for Parental Distress, Difficult Child, and Parent-Child Dysfunctional Interaction. More behavior problems were correlated with more parenting stress. Also, a significant negative correlation was found between TPF and $\mathrm{BC}$ scores, indicating that children exhibiting more TPF were more likely to also exhibit less $\mathrm{BC}$ and children exhibiting more $\mathrm{BC}$ were more likely to also exhibit less TPF.

\subsection{Analysis of Variance (ANOVA)}

Analysis of Variance (ANOVA) procedures were conducted to assess whether parents of children with high TPF scores $(\mathrm{TPF} \geq 60)$ and parents of children with high $\mathrm{BC}$ scores $(\mathrm{BC} \geq 65)$ exhibited significantly different levels of parenting stress (Table 3). Parenting stress levels were found to be significantly different between the two groups of parents. Parents with children who demonstrated more protective factors, as indicated by the TPF score (TPF $\geq 60$ ), exhibited significantly lower levels of parenting stress.

Table 3. ANOVA for high total protective factors group $(n=13)$, high behavior concerns group $(n=22)$, and parenting stress total score

\begin{tabular}{llllll}
\hline Variables & $\mathrm{M}$ & $S D$ & $\mathrm{df}$ & $\mathrm{F}$ & $\mathrm{p}$ \\
\hline Parenting Stress Total & & & 1 & 37.8 & $<.001^{* *}$ \\
$\quad$ High TPF & 53.6 & 10.9 & & & \\
$\quad$ High BC & 94.3 & 22.2 & & & \\
$\quad$ Error & & & 33 & & \\
$.05 ; * * \mathrm{p}<.01$ & & & & &
\end{tabular}

ANOVA procedures were also conducted to investigate whether parents with clinically significant levels of parenting stress (PSI-SF Total Raw Score $\geq 90$ ) and parents with non-clinical levels of parenting stress (PSI-SF Total Raw Score $\leq 89$ ) had children with different levels of protective factors and behavior concerns (Table 4). Significant differences were found between the two groups. Parents exhibiting non-clinical levels of parenting stress had children with significantly higher TPF scores compared to parents exhibiting clinical levels of parenting stress. Significant differences were also found for BC scores. Parents exhibiting non-clinical levels of parenting stress had children with significantly lower BC scores compared to parents exhibiting clinical levels of parenting stress.

Table 4. ANOVA for clinical $(n=17)$ and non-clinical $(n=59)$ parenting stress scores, total protective factors, and behavior concerns

\begin{tabular}{llllll}
\hline Variables & $\mathrm{M}$ & $S D$ & $\mathrm{df}$ & $\mathrm{F}$ & $\mathrm{p}$ \\
\hline Total Protective Factors & & & 1 & 13.7 & $<.001^{* *}$ \\
$\quad$ Clinical & 42.5 & 10.1 & & & \\
$\quad$ Non-Clinical Stress & 52.2 & 10.3 & & & \\
$\quad$ Behavior Concerns & & & 1 & 15.5 & $<.001^{* *}$ \\
$\quad$ Clinical & 65.4 & 7.5 & & & \\
$\quad$ Non-Clinical Stress & 55.6 & 9.4 & & & \\
Error & & & 74 & & \\
\hline .05;** $\mathrm{p}<.01$ & & & &
\end{tabular}

\section{Discussion}

This is the first study to examine the association between parenting stress and children's protective factors in a population that was considered behaviorally at-risk. Results of the study suggested strong associations between parenting stress, children's protective factors, and behavior concerns. As predicted, less parenting stress was related to more protective factors. Results were also consistent with past research, with elevated parenting stress 
found to be associated with behavior problems (e.g., Barry et al., 2005; Shaw et al., 1998).

The significant negative correlation between parenting stress and protective factors was slightly stronger than the significant positive correlation between parenting stress and behavior concerns. Two previous studies that have examined prosocial behaviors and parenting stress found parenting stress was overall more strongly related to children's social competence compared to behavior problems, as reported by teachers (Anthony et al., 2005; Mistry et al., 2002). The current study found similar results and extends these findings, given that children' protective factors were parent-reported, rather than teacher-reported, and were examined in relation to parenting stress levels.

The significant positive correlation between parenting stress and behavior problems in this study was also consistent with previous research. Studies examining children's behavior problems and parenting stress across time have found significant correlations between these variables (Shaw et al., 1998; Williford et al., 2007). Similarly, in the current study, reports of elevated parenting stress predicted more behavior problems. Although these studies were methodologically different given the longitudinal nature of the previous study designs, the current study found similar results.

One important finding that emerged from the study was the significant differences in parenting stress between parents of children exhibiting more protective factors and elevated behavior concerns. One explanation could be viewed from the Qi and Kaiser's modified transactional model (2000). These predicted correlations could possibly have emerged given that primary caregivers, mostly parents in this case, self-reported their levels of stress and behaviors in their children. Given that parenting stress is not a completely isolated construct, and may be prone to a "spillover" effect from other life stressors, it could be argued that parents with less stress related to parenting may be experiencing fewer life stressors compared to parents with high levels of parenting stress. With less stress, parents may view hassles related to parenting less negatively and may have more positive interactions with their child. This, in turn, may positively impact the way in which the child behaves, thus affecting a parent's perception of behavior. On the other hand, parents experiencing more parenting stress are likely to have more negative interactions with their child and more inconsistent parenting strategies, which may exacerbate problematic behaviors (Crnic et al., 2005). As suggested in previous studies utilizing parental reports, parental bias may play a role in the ratings of their child's behavior as well as their own stress (Barry et al., 2005; Shaw, et al., 1998).

Another important finding emerged when comparing children's protective factor and behavior concerns scores of parents with clinical levels of parenting stress and parents with non-clinical levels of parenting stress. Parents with non-clinical scores rated their children as exhibiting significantly more protective factors and significantly fewer behavior concerns compared to parents with parenting stress scores falling within the clinical range. Parents receiving scores in the clinical range on the PSI-SF suggest that the parent is at a high risk for dysfunctional parenting. This, coupled with their high ratings of behavior concerns and low ratings of protective factors, places the parent at an even higher risk of exacerbating children's behavior concerns and remaining at an elevated parenting stress level. While parenting stress has been found to be related to increased risk factors for children in previous studies (Barry et al., 2005) and lower levels of prosocial behaviors (Anthony et al., 2005) it was interesting that parenting stress falling in the normative range was associated with significantly higher protective factors for children. Despite the multiple risk factors experienced by the families in this sample, such as low-income, some children and parents had a desirable level of protective factors and average levels of behavior concerns and stress.

\subsection{Limitations and Future Directions}

Study findings are limited due to the study design, data collection methods, and issues related to sample composition. In regards to study design, a cause-effect relationship could not be established because there was no control for confounding variables. Also, the associations or differences found may be due to other factors not accounted for. According to parenting stress models, parenting stress is comprised of several other variables that were not examined in this study, which may influence or be interrelated to parenting stress levels, and are in need of further study.

Given that this was an exploratory study, more sophisticated methods should be employed to further investigate this important topic. Future research could examine the predictive nature parenting stress and protective factors have on one another. Similar to studies examining the changes in parenting stress and behavior problems in children over time (e.g., Williford et al., 2007), research could examine changes in parenting stress and protective factors in children over time. This would illustrate whether initial parenting stress levels or protective factors levels predict an increase, decrease, or maintenance of the other. 
Research could also examine the cause and effect relationship of these processes. By randomly assigning parents into groups where their children either receive a behavioral intervention to improve protective factors or do not receive a behavioral intervention to improve protective factors, a measurement of parenting stress levels could be used to suggest whether increasing protective factors in children affects a decrease in parenting stress levels. Alternatively, parents could be randomly assigned to receive a parenting stress intervention and measure children's level of protective factors following implementation.

Along the same lines, future research could examine additional parenting variables, including parenting stress, and its' link to children's protective factors using more advanced statistical analyses. For example, these relationships could be investigated with regression analyses, similar to Barry and colleagues' (2005) study of parenting stress and behavior problems. Longitudinal studies and hierarchical linear modeling could also be conducted to examine parenting stress and its impact on protective factors over time, similar to the method used by Williford and colleagues (2007).

A limitation related to data collection is the amount and type of questionnaires administered. Only one questionnaire assessing for protective factors and behavior concerns and one questionnaire assessing for parenting stress was used. Ratings of children's behavior and parenting stress were completed only by parents, which may have influenced or biased the way questionnaires were completed. Parental perceptions of their own stress may certainly affect the way in which parents rate their children's behavior, however it may also be the case that children's positive or negative behavior influences parents' perception of their parenting stress level.

Given that parents of children with more behavior problems are more likely to rate higher levels of stress (Shaw et al., 1998), it would be important to include multiple methods in future investigations of parenting stress and children's protective factors, such as observations in the home and classroom as well as reports from multiple informants. Ratings from other informants such as teachers may help strengthen future studies by supplementing parent perceptions of behavior that could be influenced by parental bias, similar to the methodology used in Barry and colleagues' study (2005). Also, including comprehensive questionnaires and additional questionnaires that include more domains of protective behaviors, behavior problems, and parenting stress may also take into consideration confounding variables.

In regards to the sample composition, the low sample size and the nature of the convenience sample used in the study would make it difficult to generalize these findings to other parents who have children enrolled in Head Start. Recruited participants were from a convenience sample given that the project coordinator and these Head Start programs have engaged in collaborative efforts in the past. The Head Start programs that were given fliers to distribute were not randomly chosen, rather they were chosen by the Head Start project coordinator based on location. It would help strengthen future investigations of parenting stress and protective factors in Head Start children by recruiting a larger number of participants from various geographic areas.

Although those enrolled in Head Start are low-income families, generalizing these findings to other low-income families is inappropriate. Parents of children choosing to enroll their child in Head Start may be different than parents who do not access these services. Therefore, future research should examine low-income parents and children that are not enrolled in Head Start. It would also be interesting to examine the different levels of protective factors, behavior concerns, and parenting stress levels of families attending Head Start and those not attending Head Start to examine whether Head Start may play a role in facilitating protective factors and in turn, reduce parenting stress.

Despite the study's limitations, this study was the first in examining the association between parenting stress and children's protective factors, in a population that was considered behaviorally at-risk. Given what we know about accumulative risk factors, it is important to identify and target protective factors for children and parents in prevention and early intervention efforts. Parenting stress is a factor that impacts and influences both parent and child behavior and as this study illustrated, a factor that is associated with protective and risk factors. Therefore, parenting stress is an essential component requiring further investigation in prevention and interventions efforts for the promotion of healthy social-emotional development for young children.

\subsection{Implications for Practice}

Much effort has been directed at examining factors that are associated with increased risk for young children and the development of behavior problems. The significant associations between protective factors and parenting stress in the current study warrants further investigation, however, it may be valuable to consider parents' stress levels when working with young children. Given the literature that suggests higher levels of parenting stress may be a risk factor for children and the current findings, it may be beneficial to provide education to parents to help them better understand their own child. For example, parenting stress literature has suggested that information 
about child development, child behavior, reasonable expectations for a young child, and becoming more sensitive to a child's needs may help reduce parents' stress and negative perceptions they have about their child.

Just as it may be beneficial to consider parents' stress when working with children, it is also important to consider children's strengths. Protective factors are malleable and can be enhanced for children to help improve their resilience to stressors. Increased awareness as to what parent factors may be associated with children's protective factors may help clinicians understand what these factors are and be thinking about how to address them in practice.

Although the current study was exploratory in nature and no causal relationship could be established, the results could be used to help clinicians, teachers, and providers consider parenting stress and other parenting variables that may positively or negatively impact children's behavior during the assessment and intervention process. It is important to consider not only the child's functioning, but the family's functioning as well. Considering what strengths parents have may facilitate a more well-rounded view of the family and ultimately the child.

\section{Acknowledgement}

We would like to acknowledge the families involved in the study and our Head Start partners. We would also like to especially thank Marta Kermiet for her support and assistance in recruiting families for this project. This work was funded in part by the Michigan Association of School Psychologists Research Grant and the Michigan State University Research Enhancement Grant.

\section{References}

Abidin, R. R. (1995). Parenting Stress Index (3rd ed.). Odessa, FL: Psychological Assessment Resources.

Anthony, L. G., Anthony, B. J., Glanville, D. N., Naiman, D. Q., Waanders, C., \& Shaffer, S. (2005). The relationships between parenting stress, parenting behavior, and preschoolers' social competence and behavior problems in the classroom. Infant and Child Development, 14, 133-154. http://dx.doi.org/10.1002/icd.385

Barry, T. D., Dunlap, S. T., Cotton, S. J., Lochman, J. E., \& Wells, K. C. (2005). The influence of maternal stress and distress on disruptive behavior problems in boys. Journal of the American Academy of Child and Adolescent Psychiatry, 44, 265-273. http://dx.doi.org/10.1097/00004583-200503000-00011

Brinkman, T. M., Wigent, C. A., Tomac, R. A., Pham, A. V., \& Carlson, S. J. (2007). Using the Devereux Early Childhood Assessment to identify behavioral risk and protective factors within a Head Start population. Canadian Journal of School Psychology, 22, 136-151. http://dx.doi.org/10.1177/0829573507307612

Conger, R. D., Wallace, L. E., Sun, Y., Simons, R. L., McLoyd, V. C., \& Brody, G. H. (2002). Economic pressure in African American families: A replication and extension of the family stress model. Developmental Psychology, 38, 179-193. http://dx.doi.org/10.1037/0012-1649.38.2.179

Crnic, K. A., Gaze, C., \& Hoffman, C. (2005). Cumulative parenting stress across the preschool period: Relations to maternal parenting and child behaviour at age 5. Infant and Child Development, 14, 117-132. http://dx.doi.org/10.1002/icd.384

Deater-Deckard, K. (1998). Parenting stress and child adjustment: Some old hypotheses and new questions. $\begin{array}{lllll}\text { Clinical Psychology: Science } & \text { 314-332. }\end{array}$ http://dx.doi.org/10.1111/j.1468-2850.1998.tb00152.x

Guterman, N. B., Lee, S. J., Taylor, C. A., \& Rathouz, P. J. (2009). Parental perceptions of neighborhood processes, stress, personal control, and risk for physical child abuse and neglect. Child Abuse \& Neglect, 33, 897-906. http://dx.doi.org/10.1016/j.chiabu.2009.09.008

LeBuffe, P. A., \& Naglieri, J. A. (1999). Devereux Early Childhood Assessment User's Guide. Lewisville, NC: Kaplan Press.

Linver, M. R., Brooks-Gunn, J., \& Kohen, D. E. (2002). Family processes as pathways from income to young $\begin{array}{lllll}\text { children's development. } & \text { Developmental } & \text { Psychology, } & 38, & \text { 719-734. }\end{array}$ http://dx.doi.org/10.1037/0012-1649.38.5.719

Mistry, R. S., Vandewater, E. A., Huston, A. C., \& McLoyd, V. C. (2002). Economic well-being and children's social adjustment: The role of family process in an ethnically diverse low-income sample. Child Development, 73, 935-951. http://dx.doi.org/10.1111/1467-8624.00448

Qi, C. H., \& Kaiser, A. P. (2003). Behavior problems of preschool children from low-income families: A review of the literature. Topics in Early Childhood Special Education, 23, 188-216. 
http://dx.doi.org/10.1177/02711214030230040201

Raver, C. C., Blackburn, E. K., Bancroft, M., \& Torp, N. (1999). Relations between effective emotional self-regulation, attention control, and low-income preschoolers' social competence with peers. Early Education and Development, 10, 332-350. http://dx.doi.org/10.1207/s15566935eed1003_6

Reitman, D., Currier, R. O., \& Stickle, T. R. (2002). A critical evaluation of the Parenting Stress Index-Short Form (PSI-SF) in a Head Start population. Journal of Clinical Child and Adolescent Psychology, 31, 384-392. http://dx.doi.org/10.1207/S15374424JCCP3103_10

Rutter, M. (1979). Protective factors in children's responses to stress and disadvantage. In M. W. Kent \& J. E. Rolf (Eds.), Primary prevention of psychopathology: Vol 3. Social competence in children (pp. 49-72). Hanover: VT: University Press of New England.

Sameroff, A. J., \& Chandler, M. J. (1975). Reproductive risk and the continuum of caretaking causality. In F. D. Horowitz, E. M. Hetherington, \& S. Scarr-Salapatek (Eds.), Review of child development research (Vol. 4, pp. 187-244). Chicago: University of Chicago Press.

Shaw, D. S., Winslow, E. B., Owens, E. B., \& Hood, N. (1998). Young children's adjustment to chronic family adversity: A longitudinal study of low-income families. Journal of the American Academy of Child and Adolescent Psychiatry, 37, 545-553. http://dx.doi.org/10.1097/00004583-199805000-00017

Whiteside-Mansell, L., Ayoub, C., McKelvey, L, Faldowski, R. A., Hart, A., \& Shears, J. (2007). Parenting stress of low-income parents of toddlers and preschoolers: Psychometric properties of a short form of the Parenting Stress Index. Parenting: Science and Practice, 7, 27-56. http://dx.doi.org/10.1080/15295190709336775

Williford, A. P., Calkins, S. D., \& Keane, S. P. (2007). Predicting change in parenting stress across early childhood: Child and maternal factors. Journal of Abnormal Child Psychology, 35, 251-163. http://dx.doi.org/10.1007/s10802-006-9082-3 